\title{
The embryonic blood-CSF barrier has molecular elements for specific glucose transport and for the general transport of molecules via transcellular routes
}

\author{
Maryam Parvas, David Bueno
}

Departament de Genètica, Facultat de Biologia, Universitat de Barcelona, Barcelona, Catalonia, Spain.

Email: dbueno@ub.edu; parvas_maryam@yahoo.com

Received 21 June 2010; revised 12 July 2010; accepted 15 July 2010.

\section{ABSTRACT}

In vertebrates, early brain development takes place at the expanded anterior end of the neural tube, which is filled with embryonic cerebrospinal fluid (E-CSF). We have recently identified a transient blood-CSF barrier that forms between embryonic days E3 and E4 in chick embryos and that is responsible for the transport of proteins and control of E-CSF homeostasis, including osmolarity. Here we examined the presence of glucose transporter GLUT-1 as well the presence of caveolae-structural protein Caveolin1 (CAV-1) in the embryonic blood-CSF barrier which may be involved in the transport of glucose and of proteins, water and ions respectively across the neuroectoderm. In this paper we demonstrate the presence of GLUT-1 and CAV-1 in endothelial cells of blood vessels as well as in adjacent neuroectodermal cells, located in the embryonic blood-CSF barrier. In blood vessels, these proteins were detected as early as $\mathrm{E} 4$ in chick embryos and E12.7 in rat embryos, i.e. the point at which the embryonic blood-CSF barrier acquires this function. In the neuroectoderm of the embryonic blood-CSF barrier, GLUT-1 was also detected at E4 and E12.7 respectively, and CAV-1 was detected shortly thereafter in both experimental models. These experiments contribute to delineating the extent to which the blood-CSF embryonic barrier controls E-CSF composition and homeostasis during early stages of brain development in avians and mammals. Our results suggest the regulation of glucose transport to the E-CSF by means of GLUT-1 and also suggest a mechanism by which proteins are transported via transcellular routes across the neuroectoderm, thus reinforcing the crucial role of E-CSF in brain development.
Keywords: Embryonic Cerebrospinal Fluid; GLUT-1; CAV-1; Blood-CSF Barrier; CNS Development

\section{INTRODUCTION}

The microenvironment of the Central Nervous System (CNS) is decisive for neuronal development and function. After closure of the anterior neuropore, brain walls form a large and physiologically sealed cavity that encloses embryonic cerebrospinal fluid (E-CSF), a complex and protein-rich fluid involved in several crucial roles in brain anlagen growth and development [1-8]. Chick and rat E-CSF proteomes analysed at E4 or $\mathrm{HH} 23$ (following Hamburger and Hamilton, 1951) [9] and at E12.7, respectively, include molecules whose roles explain the general functions reported for this fluid [10-13], most of which is transported from the embryonic serum to the E-CSF [14]. Recently, we identified a transient blood-CSF barrier located in the brain stem lateral to the ventral midline, at the mesencephalon and prosencephalon level, between embryonic days E3 and E5 in chick embryos and $12.7-13.7$ days post-coitum in rats $[15,16]$.

This embryonic blood-CSF barrier transports proteins in a selective manner via transcellular routes [15] as well as water and ions via specific transporters, identified by the presence of aquaporins 1 and 4 (AQP1 and AQP4) and the inwardly rectifying $\mathrm{K}+$ channel (Kir4.1) [16]. Anatomically, this barrier is formed by specific blood vessels and the adjacent neuroectoderm, and, functionally, it controls E-CSF composition and homeostasis during this early stage of brain development, before the formation of functional choroid plexuses $[15,16]$. However, several questions regarding the embryonic bloodCSF barrier deserve further investigation, such as whether it also controls the transport of energetic molecules for the developing CNS, namely glucose, and the mechanism by which protein, water and ions are transported across the neuroectoderm. In this regard, AQP1, 
AQP4 and Kir4.1 have been identified solely in the blood vessels of the embryonic blood-CSF barrier, but not in the adjacent neuroectoderm [15], and proteins following transcellular routes have been visualized within the neuroectoderm [16].

Therefore, the analysis of the anatomical location of the glucose transporter GLUT-1 and the caveolae-structural protein Caveolin1 (CAV-1), both present in the adult blood-brain barrier (BBB) [17-19], for transcellular protein, water and ion transport, may provide new data on the embryonic blood-CSF barrier. CAV-1 (21-24 kDa), a member of the caveolin family of proteins, is the main structural component of caveolae, which are 50- to $100-\mathrm{nm}$ vesicular invaginations of the plasma membrane that are involved in transcellular molecular transport, as well as in cell adhesion and signal transduction. Endothelial cells show the highest expression of CAV-1 [20], which is also found in the human BBB-provided vessels [21].

GLUT-1, an isoform of the glucose transporter (42-55 $\mathrm{kDa}$ ), is highly expressed by BBB microvessels and is localized in the endothelial plasma membrane but absent from circumventricular organs, which lack BBB properties [22-24]. Since glucose supports most cerebral functions, the glucose requirements of the brain are high [22]. Thus, GLUT-1 plays a major role in brain metabolism since it is responsible for more than $90 \%$ of the glucose transport through the BBB. Thus, GLUT-1 immunoreactivity has been detected in the neuroectoderm of developing brains of mice and rats from E9; however, it has been described that this expression progressively reduces as development proceeds, being finally restricted to intraneural capillaries [25-27]. Thus, according to Harik et al. [26], GLUT-1 immunoreactivity in rat neuroectoderm is no longer detected at E16, and according to Bauer et al. [27] this transporter is not detected in this tissue from E11 onwards in mice. These observations may reflect interspecific differences.

Here we addressed these two BBB-specific markers to further analyse some of the molecular features of the embryonic blood-CSF barrier in avian (chick) and mammal (rat) in more detail, i.e. the existence of other known transporters as GLUT-1 and of caveolae vesicles. We demonstrate the presence of glucose transporter (GLUT-1) within the same blood vessels that exhibit barrier properties for proteins, water and ions, as visualized by AQP1 co-detection, as well as in the transporting neuroectoderm adjacent to them. We also show that CAV-1 is also present in both the neuroectodermal and the endothelial cells that fulfil this barrier function, as shown by AQP1 co-detection. Our results contribute to delineating the extent to which the embryonic bloodCSF barrier controls E-CSF composition and homeosta- sis and also elucidate the mechanism by which molecules are transported across the neuroectoderm. The findings reported here reinforce the crucial role of E-CSF in brain development.

\section{MATERIAL AND METHODS}

\subsection{Obtaining Chick and Rat Embryos}

Fertile chicken eggs (Gallus gallus; White-Leghorn strain) were incubated at $38^{\circ} \mathrm{C}$ in a humidified atmosphere to obtain embryos at the desired developmental stage, i.e. at E3 (HH20), E4 (HH23) and E5 (HH26) (E is for embryonic day from the beginning of incubation; $\mathrm{HH}$ is for Hamburger and Hamilton developmental stages [9], as described in [24]. Rats (Rattus norvegicus; Wistar strain) were mated naturally. The morning of the appearance of a vaginal plug was designated as embryonic day 0.5 (E0.5). Embryos at stages E12.7 and E13.7 were dissected out from rats previously killed by $\mathrm{CO}_{2}$ asphyxia. Local animal welfare regulations were followed.

\subsection{SDS-Polyacrylamide Gel Electrophoresis and Western Blot Analysis}

SDS-polyacrylamide gel electrophoresis (SDS-PAGE) was performed under denaturing conditions following the method described by Laemmli (1979) [28], with a Miniprotean II electrophoresis system (BioRad). Samples were obtained by homogenisation of brain stem tissues from dissected chick embryos obtained at E5, and from dissected rat embryos obtained at E13.7. Protein extracts from the dorsal mesencephalon were used as negative controls. At least five embryos were pooled for each stage, and the experiment was performed four times from separate protein collections.

Molecular mass standards of high and low range (BioRad) were also used. SDS-PAGE was performed in a discontinuous buffer system for $40 \mathrm{~min}$ at 100 volts. Proteins were then electrotransferred (Trans-Blot Transfer System, BioRad) from the SDS-PAGE to activated nitrocellulose membrane (Hybond-N) for $1 \mathrm{hr}$ at 100 volts, using a basic transfer buffer and following standard protocols. Proteins were immunodetected as described in [15]. Welfare regulations were followed.

\subsection{PCR}

PCR analysis was performed on brain stems from chick embryos at E5 and rat embryos at E13.7 dissected under a dissecting microscope, and also on dorsal mesencephalon as the negative control. The total mRNA was purified by the RNeasy Mini Kit for RNA extraction (Qiagen). The primers used are shown in Table 1. A regime consisting of $94^{\circ} \mathrm{C}$ for $2 \mathrm{~min}$ ( 1 cycle), then 35 cycles of $94^{\circ} \mathrm{C}$ for $1 \mathrm{~min}, 48^{\circ} \mathrm{C}$ for $30 \mathrm{~s}$, and $72^{\circ} \mathrm{C}$ for 45 $\mathrm{s}$, followed by a single terminal extension step $\left(72^{\circ} \mathrm{C}\right.$ for 
Table 1. Primers used for PCR analysis.

\begin{tabular}{|c|c|c|c|}
\hline Gene & $\mathrm{F} / \mathrm{R}$ & Sequence ( $5^{\prime}$ to $\left.3^{\prime}\right)$ & bp \\
\hline \multicolumn{4}{|c|}{ Chick } \\
\hline \multirow{2}{*}{ GLUT-1 } & $\mathrm{F}$ & CACTGTTGTTTCGCTCTTCG & \multirow{2}{*}{316} \\
\hline & $\mathrm{R}$ & AATGTACTGGAAGCCCATGC & \\
\hline \multirow{2}{*}{ CAV-1 } & $\mathrm{F}$ & GCCCAATAACAAGATGATGGC & \multirow{2}{*}{410} \\
\hline & $\mathrm{R}$ & GGATGCTGCACACCTTGC & \\
\hline \multirow{2}{*}{ B-Actin } & $\mathrm{F}$ & CTACAATGAGCTGCGTGTGGC & \multirow[t]{2}{*}{275} \\
\hline & $\mathrm{R}$ & CAGGTCCAGACGCAGGATGGC & \\
\hline \multicolumn{4}{|c|}{ Rat } \\
\hline \multirow{2}{*}{ GLUT-1 } & $\mathrm{F}$ & GCCTGAGACTTGAAAGAAC & \multirow{2}{*}{248} \\
\hline & $\mathrm{R}$ & CTGCTTAGGTAAAGTTACAGGAG & \\
\hline \multirow{2}{*}{ CAV-1 } & $\mathrm{F}$ & GACTTTGAAGATGTGATTGC & \multirow{2}{*}{273} \\
\hline & $\mathrm{R}$ & AGATGGAATAGACACGGCTG & \\
\hline \multirow{2}{*}{ B-Actin } & $\mathrm{F}$ & CTACAATGAGCTGCGTGTGGC & \multirow{2}{*}{275} \\
\hline & & 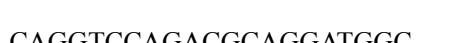 & \\
\hline
\end{tabular}

$5 \mathrm{~min}$ ) on an Eppendorf Mastercycler was used for all primer sets. Actin was used as a positive control. Each PCR experiment was performed with four separate RNA-cDNA collections from five specimens for each stage. 100bp standards were used (Quimigen). Welfare regulations were followed.

\subsection{Immunohistochemistry}

To detect CAV-1 and GLUT-1 expression, chick embryos at E3 (HH20), E4 (HH23) and E5 (HH26) and rat embryos at E12.7 and E13.7 were processed as described in [1]. These embryos were incubated with the corresponding primary antibodies: rabbit anti-GLUT1 (Millipore, $\mathrm{AB} 1340$ ) at 1/500; and rabbit anti-CAV1 (Beckton, $\mathrm{C} 13630)$ at $1 / 500$. Anti-rabbit conjugated to Alexa-488 at $1 / 500$ (Molecular Probes) was used as the secondary antibody. To simultaneously detect the area of GLUT-1 and $\mathrm{CAV}-1$ expression with respect to the previously described blood vessels involved in the blood-CSF, several sections were immunostained with rabbit anti-CAV1 or rabbit anti-GLUT1 and rabbit anti-AQP1 (Alpha Diagnostic), and the antibodies were then sequentially detected with goat anti-rabbit conjugated to Alexa-488 at 1/500 (Molecular Probes) for anti-AQP1 and goat anti-rabbit conjugated to Alexa-568 at 1/500 (Molecular Probes) for anti-GLUT1 and anti-CAV1. Half-mount embryos cut sagittally were also stained for GLUT-1 and CAV-1 expression.
Immunostained sections were counterstained with phalloidin-TRITC at 1/2000 (Sigma, P1951) and/or with TOTO-3 at $1 / 1000$ (Molecular Probes, T3604) in the presence of 1\% Rnase (Sigma, R6513) as described in [15]. Photomicrographs were taken using a confocal microscope (Olympus) or with a dissecting microscope equipped with epifluorescence (Leica MZ16F), and were assembled with Photoshop software.

\section{RESULTS}

Immunochemical methods were used to determine the localisation of the caveolae-structural protein CAV-1 and the glucose transporter protein GLUT-1 in the head region of chick and rat embryos at early developmental stages. Examination of the head region included the area from the brachial arches level (i.e. approximately from the $5^{\text {th }}$ somite) to all cephalic vesicles, thus comprising the entire brain primordium neuroectoderm as well as all surrounding tissues, i.e. the mesenchyma including the blood vessels within it, and the ectoderm. This area was selected on the basis of previous studies, in which blood vessels located in the brain stem lateral to the ventral midline, at the mesencephalon and prosencephalon level, were reported to form a blood-CSF barrier for proteins, water and ions.

Chick embryos were examined from E3 (HH18) to E5 (HH25), that is to say, just after closure of the anterior neuropore and before the initiation of neuroectodermal cavitation. Neither CAV-1 nor GLUT-1 was detected in E3 embryos (data not shown). In contrast, at E4, both CAV-1 and GLUT-1 immunostaining was detected in the endothelial cells of some blood vessels immersed in the mesenchyma in the brain stem, lateral to the ventral midline, at the mesencephalon and prosencephalon level, and close to the neuroectoderm. GLUT-1 was also expressed in the neuroectoderm close to these blood vessels at both E4 and E5. However, CAV-1 expression in the neuroectoderm was not detected at E4 but shortly thereafter. The number of CAV-1 and GLUT-1 immunoreactive blood vessels and the intensity in the adjacent neuroectoderm increased in this region from E4 to E5, as development proceeded (Figure 1(a), (b)), and they were not clearly detected in other neuroectodermal areas of the head region. At these two stages, neither CAV-1 nor GLUT-1 expression were detected in any other blood vessel or vascular sprout of the head region (Figure 1(b)).

To check whether blood vessels expressing CAV-1 and GLUT-1 were the same as those involved in the previously reported embryonic blood-CSF barrier, double immunostaining with anti-CAV1 or alternatively antiGLUT1 and with anti-AQP1 was performed (Figure 1(c)). AQP1 was chosen as it had previously been reported to 


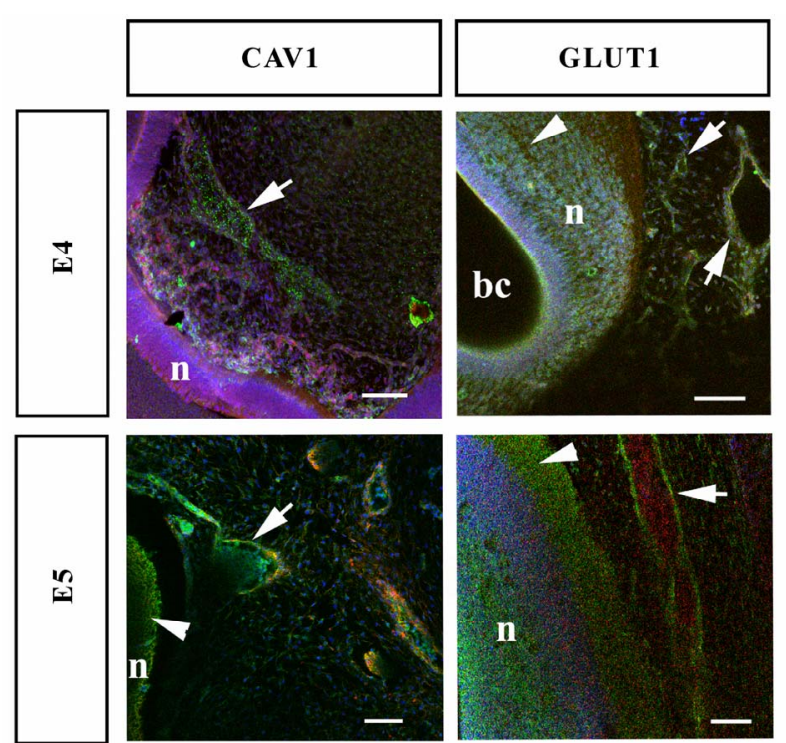

(a)
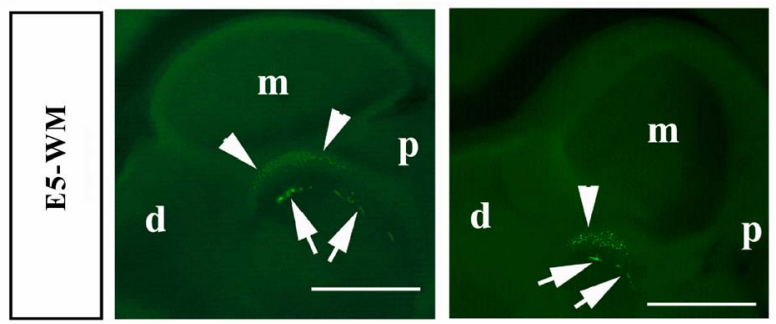

(b)

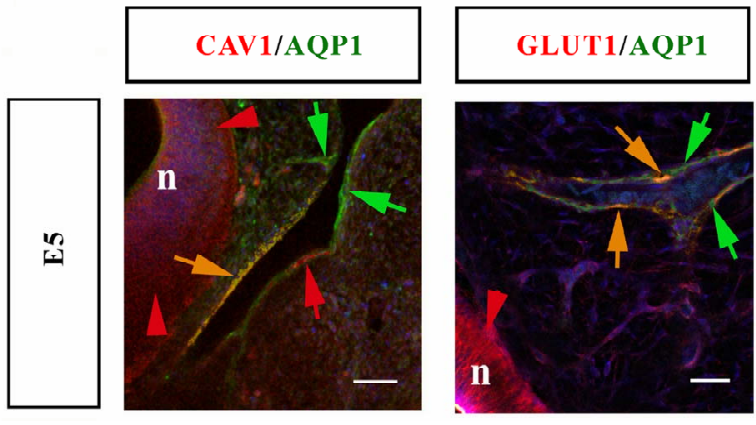

(c)

Figure 1. Immunohistochemical localisation of CAV-1 and GLUT-1 in the head process of chick embryos at E4 and E5. A and $\mathrm{C}$ are merged confocal images $(50 \mu \mathrm{m}$ thick) of $200 \mu \mathrm{m}$ vibratome sections. $\mathrm{B}$ is half-mount heads seen under a dissecting microscope. Note the immunoreactive blood vessels and vascular sprouts immersed within the parenchyma (arrows) and the staining within the neuroectoderm (arrowheads). In C), green arrows are for AQP1 green immunostaining; red arrows and arrowheads are for CAV-1 or GLUT-1 red immunostaining; and orange arrows are for areas simultaneously immunostained for AQP1 and CAV-1 or GLUT-1, which are seen in orange after merging. Nuclear counterstaining is shown in blue and cellular counterstaining in red (except in B). Scale bars: A: 0.1 $\mathrm{mm}$; B: $1 \mathrm{~mm}$; C: $0.25 \mathrm{~mm}$. Abbreviations: bc, brain cavity; $\mathrm{d}$, diencephalon; $\mathrm{m}$, mesencephalon; $\mathrm{n}$, neuroectoderm; $\mathrm{p}$, prosencephalon. be expressed in the same blood vessels fulfilling the blood-CSF function for proteins, and because its expression coincides with other molecular markers of this barrier, namely AQP4 and Kir4.1. Embryos, which were examined at E5, showed the presence of CAV-1 and AQP1 or GLUT-1 and AQP1 in the same blood vessels. Certain areas of these blood vessels expressed both molecules (CAV-1/AQP1 or GLUT-1/AQP1) simultaneously while others expressed only one of them. However, these molecules were always located in the brain stem, lateral to the ventral midline, at the mesencephalon and prosencephalon level.

To extrapolate the avian data to a mammalian system, we also examined the localisation of CAV-1 and GLUT-1 in rat embryos from E12.7 to E13.7, as these developmental stages are equivalent to those examined in chick embryos with respect to brain development. Thus, these stages correspond to the period between the closure of the anterior neuropore and the initiation of neuroectodermal cavitation, coincide with the starting period of maximum anterior CNS neurogenesis, and occur before the functional embryonic choroid plexus is formed. This strategy enabled us to determine whether mammals have a similar area with blood-CSF barrier functions.

In rat embryos, CAV-1 and GLUT-1 were expressed at both E12.7 and E13.7 in the endothelial cells of some blood vessels located within the mesenchyma in the brain stem, lateral to the ventral midline, at the mesencephalon and prosencephalon level, as well as in some vascular sprouts penetrating the neuroectoderm. With respect to neuroectodermal cells close to these blood vessels, GLUT-1 was detected at both E12.7 and E13.7. However, as in chick embryos CAV-1 expression was not detected at E12.7 but shortly thereafter (Figure 2). Again, at these two stages, CAV-1 and GLUT-1 expression was not detected in any other blood vessel or vascular sprout of the head and immunostaining within the neuroecftoderm was not clearly detected in other neuroectodermal areas of the head region.

Finally, to check the specificity of the immunodetection of CAV-1 and GLUT-1 in the brain stem of chick and rat embryos, we performed Western blot and PCR analysis on brain stem protein and RNA extracts respectively, obtained from E5 chick and E13.7 rat embryos, as well as on extracts from the dorsal mesencephalon, as a negative control. The corresponding proteins and RNAs were detected in rat and chick brain stem extracts at the expected molecular mass and nucleotide length respectively (Figure 3).

\section{DISCUSSION}

In adults, CSF composition and homeostasis is controlled 


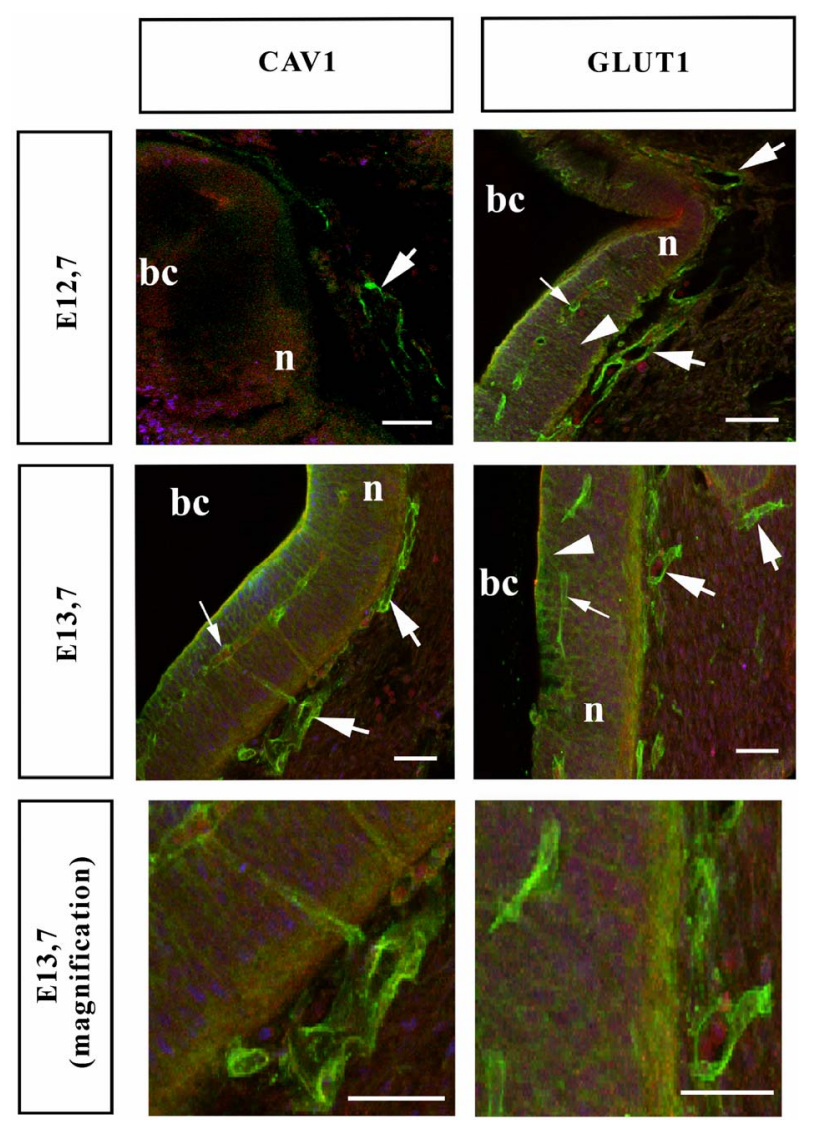

Figure 2. Immunohistochemical localization of CAV-1 and GLUT-1 in the head process of rat embryos at E12.7 and E13.7. Merged confocal image $(50 \mu \mathrm{m}$ thick) of $200 \mu \mathrm{m}$ vibratome sections. Note the immunoreactive blood vessels immersed within the parenchyma (thick arrows), the immunoreactive vascular sprouts within the neuroectoderm (thin arrows) and the staining within the neuroectoderm (arrowheads), in green. Nuclear counterstaining is shown in blue and cellular counterstaining in red. Last column shows a magnified area from the images of the middle column. Scale bars: $0.1 \mathrm{~mm}$. Abbreviations: bc, brain cavity; n, neuroectoderm.

by the choroid plexus and, to some extent, by BBB-provided vessels within the brain. The relevance of bloodto-brain and blood-to-CSF barriers for CNS development is beginning to be elucidated, although little is known about early embryos. Recently, we demonstrated the presence of blood-barrier-forming vessels in chick and rat embryos, located in the brain stem, lateral to the ventral midline, at the mesencephalon and prosencephalon level. The barrier properties develop between E3 (HH18) and E4 (HH24) in chick embryos and at E12.7 in rat embryos, the equivalent developmental stage in terms of brain vesicle formation. The blood vessels exhibiting these properties, in conjunction with the neuroectoderm adjacent to them, provide an effective blood-CSF barrier for proteins as early as E4 in chick embryos [15], i.e. just after the closure of the neuropores,

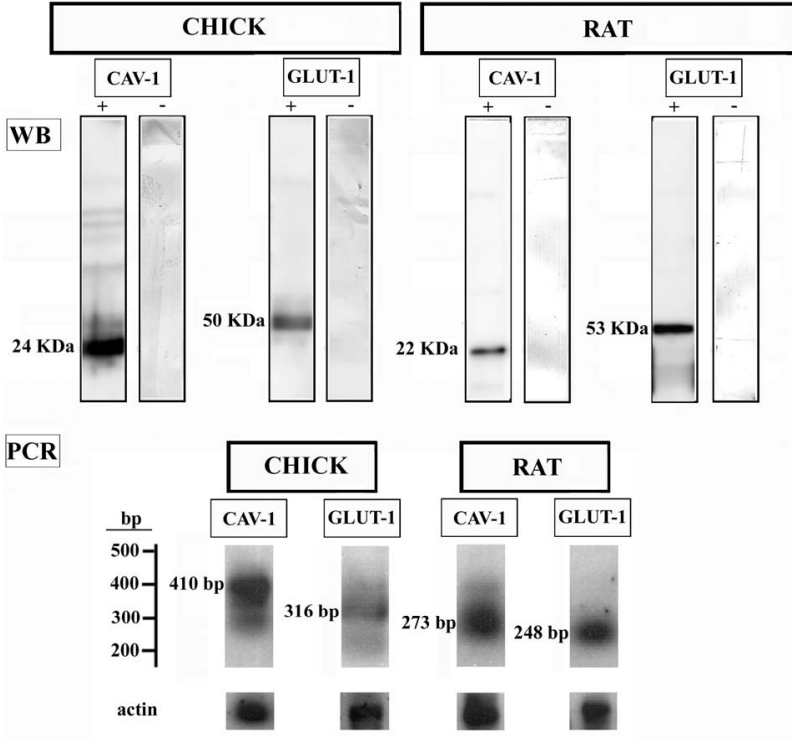

Figure 3. Western blot and PCR analysis of CAV-1 and GLUT-1 in the brain stem $(+)$ of chick and rat embryos at E5 and E13.7 respectively. Negative controls (-) for Western blot were made from the homogenized dorsal mesencephalon of chick and rat embryos at E5 and E13.7 respectively. Actin controls for PCR are also shown.

at a developmental stage at which the neural progenitor cells forming the brain wall exhibit both intense proliferation and the initiation of a period characterized by a high rate of neurogenesis.

Moreover, we also showed that channels for water (AQP1 and AQP4) and ion transport (inwardly rectifying $\mathrm{K}+$ channel Kir4.1) were present in the same blood vessels that carry out barrier functions for blood-CSF protein transport. This observation thus supports the hypothesis that these blood vessels, together with the adjacent neuroectoderm, regulate E-CSF composition and homeostasis [16]. Interestingly, this area does not coincide with the zones from which the adult choroid plexuses form in a subsequent developmental stage [24]. Later in development, during foetal stages, as well as in adults, choroid plexuses and BBB-provided vessels express a wide variety of specific transporters for a broad spectrum of molecules, ranging from proteins to glucose, amino acids, water and ions.

Our findings show that GLUT-1, a glucose transporter, and CAV-1, which is present in caveolae transcellular transport in the adult BBB, are also in blood vessels located in the embryonic blood-CSF barrier in chick and rat embryos at early stages of brain development. That is to say, they are present in blood vessels located in the brain stem, lateral to the ventral midline, at the mesencephalon and prosencephalon level, where protein transport from embryonic serum to the E-CSF and vice versa has been described [13]. Moreover, the presence of 
blood vessels expressing water and ion channel proteins (i.e. AQP1, AQP4 and Kir4.1) has been reported. Thus, double immunostained sections of chick embryos have shown that GLUT-1 and CAV-1 are expressed in the same blood vessels expressing AQP1, which in turn are those that express CAV-4 and Kir4.1, as previously reported [16]. On the basis of our results and those from previous papers, we propose that in mammals these molecules may also be expressed in the same blood vessels, namely those involved in the blood-CSF barrier.

Moreover, GLUT-1 was also detected in the neuroectoderm involved in the embryonic blood-CSF barrier, thereby suggesting that glucose destined for developing brain cells is transported from the blood to the E-CSF by this glucose transporter. Of note, GLUT-1 was not detected in any other location of the brain stem, thus indicating the relevance of this barrier area for E-CSF composition control and homeostasis. Previous reports on GLUT-1 expression [25-27] indicate that this glucose transporter is expressed in mammals (mice in rat) from very early stages of brain development throughout the entire brain neuroectoderm, but that its expression decreases as development proceeds, becoming finally undetectable. In this regard, Dermietzel et al. [25] showed that the gradual decrease in GLUT-1 expression is not homogeneous in the neuroectoderm, and that this glucose transporter becomes progressively restricted to some brain areas, including that involved in the blood-CSF barrier, as reported in this paper.

Conversely, AQP1 and AQP4 for water transport, and Kir4.i for ion transport are present solely in these blood vessels [16], but not in the adjacent neuroectoderm. These observations therefore raise questions regarding the mechanisms by which they are transported through this tissue to reach the E-CSF. In this regard, protein transport has been reported to be transcellular [15], but no transcellular transporting structures have been described. Our results demonstrate that CAV-1 is present in the blood vessels and the neuroectoderm forming the embryonic blood-CSF barrier. As caveolae are involved in transcellular transport, we propose that these are the structures by which proteins are transported from blood to E-CSF and vice versa. In addition, as caveolae may also transport water and ions alongside proteins, and neither water nor ion transporters have been identified in the embryonic blood-CSF barrier neuroectoderm, it is possible that these molecules, when outside the blood vessels, are transported by the caveolae to the E-CSF, along with transported proteins. However, the lack of functional data do not allow us to support this hypothesis. Finally, CAV-1 was not detected in any other location of the brain stem. This observation also indicates the relevance of this barrier area for E-CSF composition control and homeostasis. It is also important to note that GLUT-1 and CAV-1 were detected at the same time as the development of protein barrier properties in chick embryos at E4, as previously reported [15].

\section{CONCLUSIONS}

Our results suggest that 1) glucose destined for the developing brain cells is transported from the blood to the E-CSF across endothelial and neuroectodermal cells that form the embryonic blood-CSF barrier via the GLUT-1 glucose transporter; 2) proteins are transported across these embryonic tissues by CAV-1 caveolae-transcellular transport; and 3) water and ions, following transport out of the blood vessels by specific transporters, are taken by CAV-1 caveolae to be transported to the E-CSF along with proteins. Taken together, our results reinforce the hypothesis of an embryonic blood-CSF barrier in both avians and mammals that controls E-CSF composition and homeostasis, i.e. protein, water, ion and glucose transport, and acts before the formation of the choroid plexuses, thus contributing to the roles ascribed to the E-CSF in early brain development.

\section{ACKNOWLEDGEMENTS}

This work was supported by a grant from the "Ministerio de Educación y Ciencia" (BFU2007-62361-FEDER) to DB. MP has a Fellowship grant from the Catalan Government (Generalitat de Catalunya-FI)

\section{REFERENCES}

[1] Alonso, M.I., Gato, A., Moro, J.A., Martin, P. and Barbosa, E. (1999) Involvement of sulfated proteoglycans in embryonic brain expansion at earliest stages of development in rat embryos. Cells Tissues Organs, 165(1), 1-9.

[2] Alonso, M.I., Gato, A., Moro, J.A. and Barbosa, E. (1998) Disruption of proteoglycans in neural tube fluid by D-xyloside alters brain enlargement in chick embryos. The Anatomical Record Part A, 252(4), 499-508.

[3] Desmond, M.E. and Jacobson, A.G. (1977) Embryonic brain enlargement requires cerebrospinal fluid pressure. Developmental Biology, 57(1), 118-198.

[4] Jelinek, R. and Pexieder, T. (1970) Pressure of the CNS in chick embryo. Folia morphologica, 2, 102-110.

[5] Miyan, J.A., Nabiyouni, M. and Zendah, M. (2003) Development of the brain: A vital role for cerebrospinal fluid. Canadian Journal of Physiology and Pharmacology, 81(4), 317-328.

[6] Gato, A. and Desmond, M.E. (2009) Why the embryo still matters: CSF and the neuroepithelium as interdependent regulators of embryonic brain growth, morphogenesis and histiogenesis. Development Biology, 327(2), 263-272.

[7] Gato, A., Moro, J.A., Alonso, M.I., Bueno, D., De La Mano, A. and Martin. C. (2005) Embryonic cerebrospinal fluid regulates neuroepithelial survival, proliferation, and neurogenesis in chick embryo. The Anatomical Record 
Part A, 284(1), 475- 484.

[8] Parada, C., Martín, C., Alonso, M.I., Moro, J.A., Bueno, D. and Gato, A. (2005) Embryonic cerebrospinal fluid collaborates with the isthmic organizer to regulate mesencephalic gene expression. Journal of Neuroscience Research, 82(3), 333-345.

[9] Hamburger, V. and Hamilton, H.L. (1951) A series of normal stages in the development of the chick embryo. Journal of Morphology, 88, 49-92.

[10] Parada, C., Gato, A. and Bueno, D. (2005) Mammalian embryonic cerebrospinal fluid proteome has greater apolipoprotein and enzyme pattern complexity than the avian proteome. Journal of Proteome Research, 4(6), 2420-2428.

[11] Parada, C., Gato, A., Aparicio, M. and Bueno, D. (2006) Proteome analysis of chick embryonic cerebro-spinal fluid. Proteomics, 6(1), 1-34.

[12] Zappaterra, M.D., Lisgo, S.N., Lindsay, S., Gygi, S.P., Walsh, C.A. and Ballif, B.A. (2007) A comparative proteomic analysis of human and rat embryonic cerebrospinal fluid. Journal of Proteomic Research, 6(9), 35373548.

[13] Parada, C., Parvas, M. and Bueno, D. (2007) Cerebrospinal fluid proteomes: From neural development to neurodegenerative diseases. Current Proteomics, 4, 89-106.

[14] Parvas, M., Rius, M. and Bueno, D. (2008) Most of the abundant protein fractions of embryonic cerebrospinal fluid are produced out of the brain anlagen. The Open Proteomics Journal, 1, 1-4.

[15] Parvas, M., Parada, C. and Bueno, D. (2008) A blood-CSF barrier function controls embryonic CSF protein composition and homeostasis during early CNS development. Developmental Biology, 321(1), 51-63.

[16] Parvas, M. and Bueno, D. (2010) The embryonic blood-CSF barrier controls E-CSF osmolarity during early CNS development. The Journal of Neuroscience Research, 88(6), 1205-1212.

[17] Rubin, L.L. and Staddon, J.M. (1999) The cell biology of the blood brain barrier. The Annual Review of Neuroscience, 22, 11-28.

[18] Frank, P.G., Woodman, S.E., Park, D.S. and Lisanti, M.P. (2003) Caveolin, caveolae, and endothelial cell function. Arteriosclerosis, Thrombosis, and Vascular Biology, 23,
1161-1168.

[19] Virgintino, D., Robertson, D., Errede, M., Benagiano, V., Taure, U., Roncali, L. and Bertossi, M. (2002) Expression of caveolin-1 in human brain microvessels. Neroscience, 115(1), 145-152.

[20] Dermeitzel, R. and Krause, D. (1991) Molecular anatomy of the blood brain barrier as defined by immunocytochemistry. In: Jeon, K.W. and Friedlander, M. Eds., International review of cytology. Academic Press, New York, 57-109.

[21] Laterra, J. and Goldstein, G.W. (1993) Brain microvessels and microvascular cells in vitro. In: Pardridge, W.M. Ed., The blood-brain barrier, Raven Press, New York, $1-24$.

[22] Pardridge, W.M. and Boado, R.J. (1993) Molecular cloning and regulation of gene expression of blood-brain barrier glucose transporter. In: Pardridge, W.M. Eds., The blood-brain barrier, Raven Press, New York, 395-440.

[23] Rahner-Welsch, S., Vogel, J. and Kuschinsky, W. (1995) Regional congruence and divergence of glucose transporters (GLUT1) and capillaries in rat brains. Journal of Cerebral Blood Flow \& Metabolism, 15, 681-686.

[24] Bellairs, R. and Osmond, M. (2005) Atlas of Chick Development. Elsevier Academy Press, London.

[25] Dermietzel, R., Krause, D., Kremes, M., Wang, C. and Stevenson, B. (1992) Pattern of glucose transporter (Glut1) expression in embryonic brains is related to maturation of blood-brain barrier tightness. Developmental Dynamics, 193, 152-163.

[26] Harik, S.I., Hall, A.K., Richey, P., Andersson, L., Lundahl, P. and Perry, G. (1993) Ontogeny of the erythroid/ HepG2-type glucose transporter (GLUT-1) in the rat nervous system. Developmental Brain Research, 72, 4149.

[27] Bauer, H., Sonnleitner, U., Lametschwandter, A., Steiner, M., Adam, H. and Bauer, H.C. (1995) Ontogenic expression of the erythroid-type glucose transporter (Glut1) in the telencephalon of the mouse: Correlation to the tightening of the blood-brain barrier. Developmental Brain Research, 86(1-2), 317-325.

[28] Laemmli, U.K, (1970) Cleavage of structural proteins during the assembly of the head of bacteriophage $\mathrm{T} 4$. Nature, 227, 680-685. 